\title{
Recycling and Wall Pumping in Long Duration Discharges on TRIAM-1M
}

\author{
M. Sakamoto, S. Itoh, K. Nakamura, H. Zushi, K. Hanada, E. Jotaki, Y. D. Pan 1), \\ S. Kawasaki, H. Nakashima
}

Advanced Fusion Research Center, Research Institute for Applied Mechanics, Kyushu University, Kasuga, Fukuoka, 816-8580 Japan

1) On leave from Southwestern Institute of Physics, 610041 China.

e-mail contact of main author: sakamoto@triam.kyushu-u.ac.jp

\begin{abstract}
Recycling and wall pumping have been studied comparing low $\left(\sim 10^{18} \mathrm{~m}^{-3}\right)$ and high $\left(\sim 10^{19} \mathrm{~m}^{-3}\right)$ density long duration plasmas in TRIAM-1M. The recycling coefficient of each plasma increases with time. There exist two time constants in the temporal evolution of the recycling coefficient. One is a few seconds and the other is about $30 \mathrm{~s}$. They may relate with characteristic times during which the physical adsorption and absorption due to the CX neutrals reach the equilibrium state, respectively. The wall pumping rates of low and high density plasmas are evaluated to be $\sim 1.5 \times 10^{16}$ atoms $\mathrm{m}^{-2} \mathrm{~s}^{-1}$ and $\sim 4 \times 10^{17}$ atoms $\mathrm{m}^{-2} \mathrm{~s}^{-1}$, respectively. The difference is caused by the difference of the total amount of the CX neutral flux with the energy of $<0.7 \mathrm{keV}$. In the ultra-long discharge ( $70 \mathrm{~min}$ ), the recycling coefficient becomes unity or more and again decreases below unity, i.e. the wall repeats a process of being saturated and refreshed. This refreshment of the wall seems to be caused by the co-deposition of Mo, which is a material of the limiter and divertor plates. In the high power and high density experiments, the wall saturation phenomenon has been observed. The discharge duration limited by the wall saturation decreases with increase in the density.
\end{abstract}

\section{Introduction}

Steady state plasma operation is one of the requirements of the future fusion reactor. In the long duration steady state plasma, various time constants (e.g. a current diffusion time, a recycling coefficient variation time, etc.) should be taken into account to control the optimized plasma, although they seriously do not affect the plasma in a short pulse discharge.

Regarding the recycling coefficient, if it increases to and above unity, the plasma density cannot be controlled as observed in one minute discharge of JET [1]. In a long pulse plasma of Tore Supra, the density became uncontrollable, that is considered to be caused by increase in an outgassing of internal elements which are located far from the plasma edge and slowly heated by the radiated power [2].

In TRIAM-1M, the ultra-long tokamak operation with the duration of longer than two hours has been demonstrated and the wall recycling and wall pumping properties in such long duration discharges have been investigated $[3,4]$. In this paper, the wall recycling and wall pumping properties, and wall saturation phenomenon are described comparing the low and high density long duration discharges.

\section{Experimental Results and Discussion}

Properties of wall recycling and wall pumping have been investigated in both $2.45 \mathrm{GHz}$ and $8.2 \mathrm{GHz}$ lower hybrid current drive (LHCD) discharges on TRIAM-1M $\left(R_{\text {major }} \sim 0.8 \mathrm{~m}, a\right.$ $\times b=0.12 \mathrm{~m} \times 0.18 \mathrm{~m})$. The experimental conditions are as follows: The working gas is hydrogen. The RF power $\mathrm{P}_{\mathrm{rf}}$ of $2.45 \mathrm{GHz} \mathrm{LH}$ wave $\left(n_{/ / \text {peak }} \sim 1.7\right)$ is $\sim 20 \mathrm{~kW}$ and that of 8.2 $\mathrm{GHz} \mathrm{LH}$ wave $\left(n_{/ / \text {peak }} \sim 1.8\right)$ is $<300 \mathrm{~kW}$. The line averaged electron density $\overline{\mathrm{n}}_{\mathrm{e}}$ is 0.1 to 0.2 


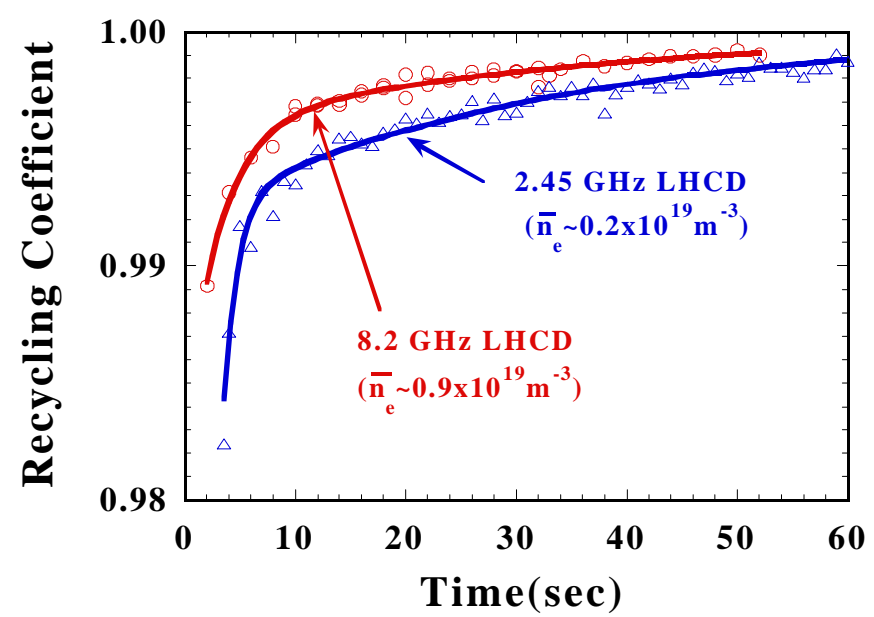

Fig.1 Time evolution of the recycling coefficients of $2.45 \mathrm{GHz} \mathrm{LHCD}$ (triangles) and $8.2 \mathrm{GHz} \mathrm{LHCD}$ (circles) plasmas.

$\times 10^{19} \mathrm{~m}^{-3}$ for $2.45 \mathrm{GHz}$ LHCD plasma and 1 to $3 \times 10^{19} \mathrm{~m}^{-3}$ for $8.2 \mathrm{GHz}$ LHCD plasma. It should be noticed that the plasma is sustained by the RF power alone. The material of the poloidal limiters and divertor plates is molybdenum and wall material is stainless steel without low $\mathrm{Z}$ material coating.

The recycling coefficient (ratio of in-flux to out-flux on the plasma surface), $R$, is obtained using a following particle balance equation;

$$
\mathrm{d} N_{\mathrm{e}} / \mathrm{d} t=\eta S_{\mathrm{g}}+(R-1) N_{\mathrm{e}} / \tau_{\mathrm{p}}
$$

where $N_{\mathrm{e}}$ is the total number of electrons, $\eta$ is fueling efficiency, $S_{\mathrm{g}}$ is gas feed rate by the piezo-electric valve, $\tau_{\mathrm{p}}$ is a particle confinement time. The plasma density is feedback controlled to keep $\mathrm{H}_{\alpha}$ line intensity at a central chord constant by the piezo-electric valve.

\subsection{Wall Recycling}

Figure 1 shows time evolutions of recycling coefficients in $2.45 \mathrm{GHz}$ and $8.2 \mathrm{GHz} \mathrm{LHCD}$ plasmas. Each recycling flux to the plasma surface is $\sim 5.4 \times 10^{18}$ atoms $\mathrm{m}^{-2} \mathrm{~s}^{-1}$ for $2.45 \mathrm{GHz}$ LHCD plasma and $\sim 5.7 \times 10^{19}$ atoms $\mathrm{m}^{-2} \mathrm{~s}^{-1}$ for $8.2 \mathrm{GHz}$ LHCD plasma, respectively. Each recycling coefficient increases with time. Solid curves are drawn using the following equation to fit the data.

$$
R(t)=1-R_{1} \exp \left(-t / \tau_{1}\right)-R_{2} \exp \left(-t / \tau_{2}\right)
$$

The values of $\tau_{1}$ and $\tau_{2}$ for $2.45 \mathrm{GHz}$ LHCD plasma are $1.3 \mathrm{~s}$ and $32 \mathrm{~s}$ and those for $8.2 \mathrm{GHz}$ LHCD plasma are $3.1 \mathrm{~s}$ and $35 \mathrm{~s}$, respectively. The fitting function above can reproduce the data very well. The recycling coefficient varies with two time constants. Shorter time constant is 1 to $3 \mathrm{~s}$ and longer one is about $30 \mathrm{~s}$.

The mechanisms of the wall pumping are mainly physical adsorption, chemical adsorption and absorption due to high energy particles. The chemical adsorption does not contribute in TRIAM-1M, since the materials of the first wall are only heavy metals. The capacity of the absorption is much higher than that of the physical adsorption. So, the shorter time constant may relate with the time during which the physical adsorption reaches an equilibrium state and the longer one may relate with the time during which the absorption of charge exchange neutrals reaches an equilibrium state. 


\subsection{Wall Pumping}

Waveforms of $2.45 \mathrm{GHz}$ LHCD discharge with the duration of $70 \mathrm{~min}$ are shown in Fig. 2. The plasma current is about $20 \mathrm{kA}$. The hydrogen gas was supplied through a piezo electric valve so as to keep the $\mathrm{H} \alpha$ line intensity at the central chord constant as shown in Figs.2 (b) and (c). The averaged gas feed rate from 10 to $40 \mathrm{~min}$ is about $1 \times 10^{17}$ atoms s${ }^{-1}$. It can be seen that the gas feed was automatically stopped several times during the discharge due to the feedback control of gas supply. It means that the recycling coefficient becomes unity or more and again decreases below unity, i.e. the wall seems to repeat a process of being saturated and refreshed. The time evolutions of the total amount of gas supply (solid line) and the particles evacuated by an external pump-unit (broken line) are shown in Fig.2 (d). From the viewpoint of the particle conservation law, the difference (indicated by " $\mathrm{B}$ " in the figure) between the two lines means the amount of the particles pumped by the wall, since the plasma density and the neutral gas pressure are kept almost constant during the discharge. The averaged wall pumping rate is about $\sim 1.5 \times 10^{16}$ atoms $\mathrm{m}^{-2} \mathrm{~s}^{-1}$. About $3 \times 10^{20}$ hydrogen atoms are pumped by the wall for $70 \mathrm{~min}$.

In such long discharge, wall condition continues to change during the discharge. For example, the temperature of the wall changes with a characteristic time of 5 to $30 \mathrm{~min}$, which depends on the distance from the cooling pipe. The radiation damage is being caused by high

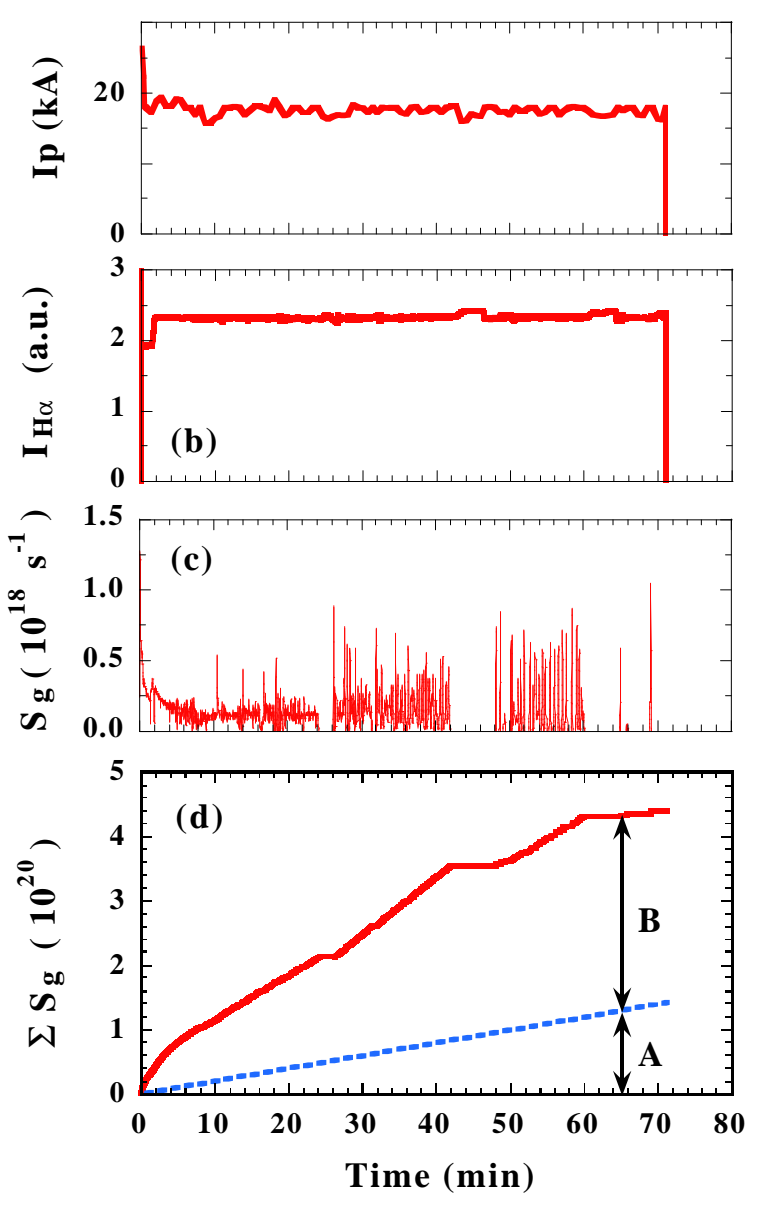

Fig.2 Time evolution of (a) plasma current,

(b) $H_{\alpha}$ line intensity, (c) gas feed rate and

(d) total amount of gas feed in $2.45 \mathrm{GHz}$

LHCD discharge.

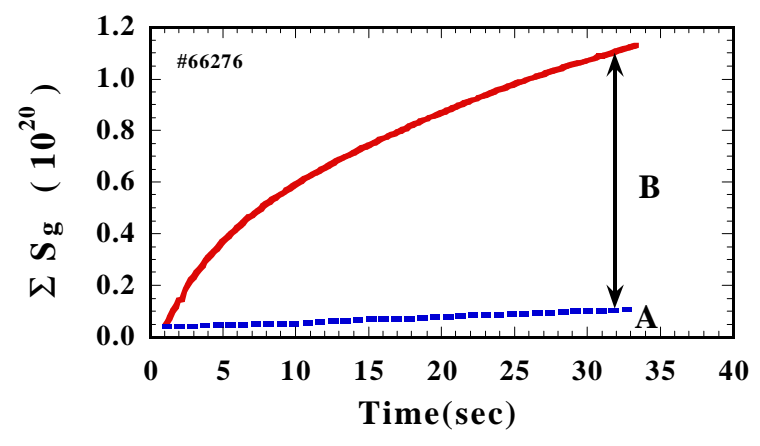

Fig.3 Time evolution of total amount of gas feed and evacuation to the external pumpunit in $8.2 \mathrm{GHz}$ LHCD discharge.

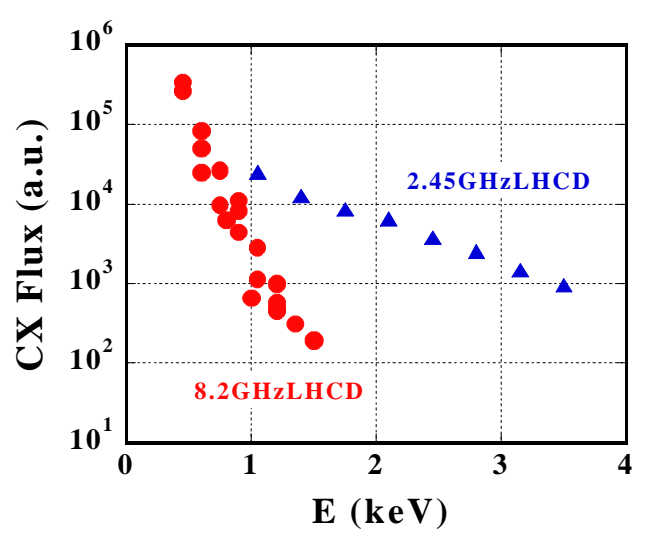

Fig.4 Energy spectra of charge exchange neutrals of $2.45 \mathrm{GHz}$ and $8.2 \mathrm{GHz} \mathrm{LHCD}$ plasmas. 
energy charge exchange (CX) neutrals and it provides a new particle-trap area. Co-deposition of in-vessel elements, so called tokamakium, also occurrs during the discharge [5]. Strong radiation damage and the tokamakium have practically been observed in TRIAM-1M [6]. The co-deposition of Mo, which is a material of poloidal limiter and divertor plates, is one candidate for the refreshment of the wall (refreshing wall) as observed in the ultra-long discharge [7,8].

Figure 3 shows the total amount of gas supply and evacuation by the external pump-unit in $8.2 \mathrm{GHz}$ LHCD discharge $\left(\mathrm{I}_{\mathrm{p}} \sim 25 \mathrm{kA}, \overline{\mathrm{n}}_{\mathrm{e}} \sim 0.8 \times 10^{19} \mathrm{~m}^{-3}, \mathrm{P}_{\mathrm{rf}} \sim 95 \mathrm{~kW}\right)$ with the duration of $33 \mathrm{~s}$. The averaged wall pumping rate per unit area is evaluated to be about $4 \times 10^{17}$ atoms $\mathrm{m}^{-2} \mathrm{~s}^{-1}$ in the same way as Fig.2(d). It is $\sim 30$ times higher in magnitude than that of $2.45 \mathrm{GHz}$ LHCD discharge, although the wall conditions just before both discharges indicated in Fig.2 and Fig.3 seem to be similar.

The temperature of SOL is approximately the same in both $2.45 \mathrm{GHz}$ and $8.2 \mathrm{GHz} \mathrm{LHCD}$ plasmas. The temperature in front of the wall is several eV. The SOL density of $8.2 \mathrm{GHz}$ LHCD plasma is $\sim 5 \times 10^{17} \mathrm{~m}^{-3}$ and it is one order higher in magnitude than that of $2.45 \mathrm{GHz}$ LHCD plasma. Such low temperature does not contribute to the wall pumping. We notice the CX neutrals for the wall pumping phenomenon. Figure 4 shows energy spectra of the CX neutrals in $2.45 \mathrm{GHz}$ and $8.2 \mathrm{GHz}$ LHCD plasmas. The CX neutral flux of $8.2 \mathrm{GHz}$ LHCD is higher than that of $2.45 \mathrm{GHz}$ below $\mathrm{E} \sim 0.7 \mathrm{keV}$ and vice versa above $\mathrm{E} \sim 0.7 \mathrm{keV}$. The difference between wall pumping rates of low and high density plasmas seems to be caused by the difference of the total amount of the CX neutral flux with the energy of $<0.7 \mathrm{keV}$.

\subsection{Wall Saturation Phenomenon}

In long duration discharge, recycling coefficient increases and reaches unity (i.e. wall saturation). As shown in Fig.2, the wall becomes saturated and refreshed in the low density LHCD discharge. In the high power and high density discharges of 8.2 GHz LHCD, however, the wall saturates and does not recover as shown in Fig.5. The RF power is $127 \mathrm{~kW}$ and the
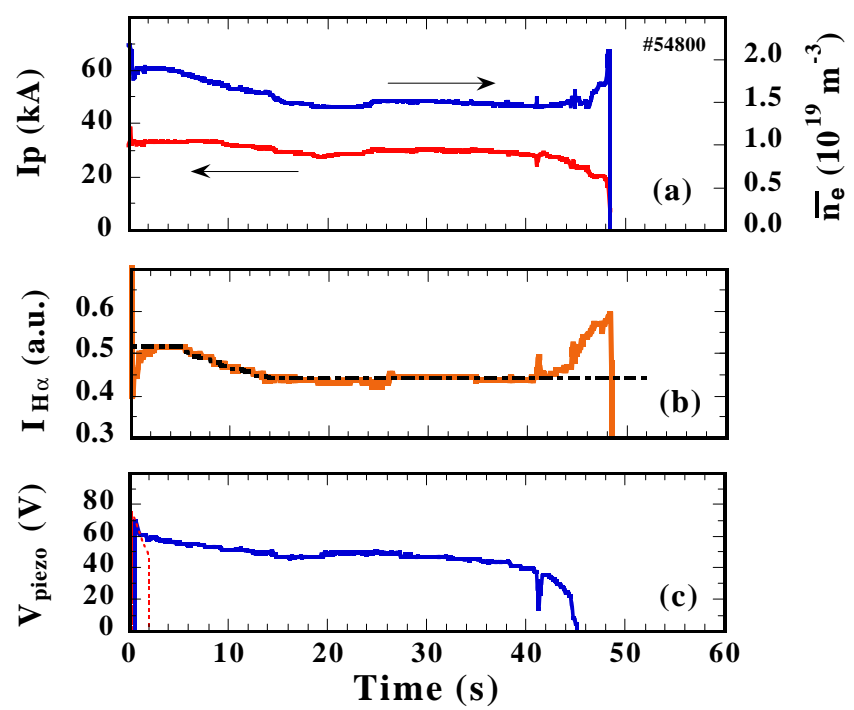

Fig.5 Time evolution of (a) plasma current and electron density, (b) $H_{\alpha}$ line intensity and reference level for feedback control (broken line) and (c) the voltage of piezo electric valves in the wall saturation discharge.

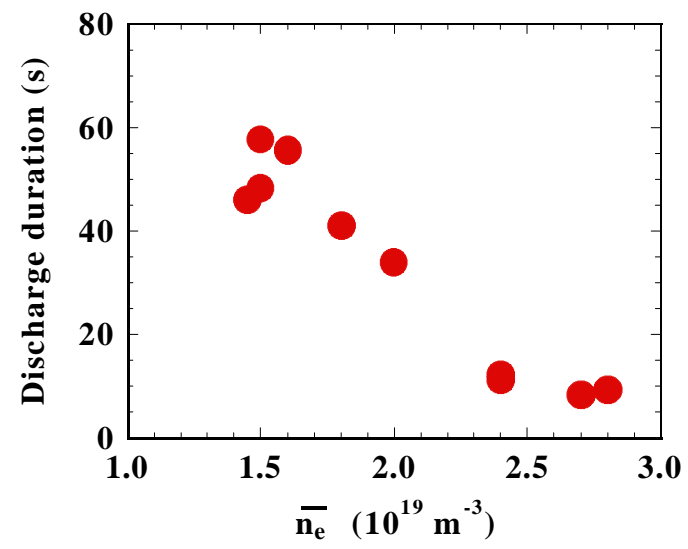

Fig.6 The restriction of discharge duration due to the wall saturation as a function of the line averaged electron density. 
plasma current is about $30 \mathrm{kA}$. The electron density is decreased from $2 \times 10^{19} \mathrm{~m}^{-3}$ to $1.5 \times 10^{19}$ $\mathrm{m}^{-3}$ to reduce the particle loading to the wall. The gas supply is well controlled till $40 \mathrm{~s}$ as shown in Fig.5(b). To decrease the density, the reference level for the feedback control is pre-programmed such as the broken curve in Fig. 5(b). The signal of $\mathrm{H}_{\alpha}$ line intensity follows the reference level and the density also follows. After $\mathrm{t} \sim 40 \mathrm{~s}$, the $\mathrm{H}_{\alpha}$ line intensity increases over the reference level and it cannot be controlled at all even by closing the piezo electric valve. The plasma current decreases due to the density increase, since the RF power is constant. At last, the plasma cannot be sustained by the RF power alone. The restriction of the discharge duration due to the wall saturation in the high power and high density experiment is shown in Fig.6 as a function of the line averaged electron density. As the density increase higher, the discharge duration becomes shorter. This dependence should be attributed to increase of particle loading (CX neutral flux) due to the plasma density increase.

\section{Summary}

Recycling and wall pumping are crucial issues for the density control of a steady state plasma. They have been studied comparing low and high density long duration plasmas in TRIAM-1M. The recycling coefficient of each plasma increases with time. There exist two time constants in the temporal evolution of the recycling coefficient. One is a few seconds and the other is about $30 \mathrm{~s}$. There is a possibility that they relate with the time during which the physical adsorption and absorption due to the $\mathrm{CX}$ neutrals reach the equilibrium state, respectively. The wall pumping rates of low $\left(\sim 0.1 \times 10^{19} \mathrm{~m}^{-3}\right)$ and high $\left(\sim 0.8 \times 10^{19} \mathrm{~m}^{-3}\right)$ density plasma are $\sim 1.5 \times 10^{16}$ atoms $\mathrm{m}^{-2} \mathrm{~s}^{-1}$ and $\sim 4 \times 10^{17}$ atoms $\mathrm{m}^{-2} \mathrm{~s}^{-1}$, respectively. The difference of the wall pumping rates is attributed to the difference of the total amount of the CX neutral flux with the energy of $<\sim 0.7 \mathrm{keV}$. In the ultra-long discharge, the recycling coefficient becomes unity or more and again decreases below unity, i.e. the wall repeats a process of being saturated and refreshed. This refreshment of the wall seems to relate to the co-deposition of Mo, which is a material of the limiter and divertor plates. In the high power and high density experiments, the wall saturation phenomenon has been observed. The discharge duration limited by the wall saturation decreases with increase in the plasma density.

\section{Acknowledgements}

The authors are grateful to Prof. Dr. D. F. Duechs and Dr. R. Behrisch for useful discussions and suggestions.

\section{References}

[1] BRUSATI, M., et al., in Controlled Fusion and Plasma Physics (Proc. 19th Eur. Conf. Innsbruck, 1992) Vol.16C, Part I, European Physical Society, Geneva (1992) 307.

[2] EQUIPE TORE SUPRA (prepared by F. Saint-Laurent), Nucl. Fusion 40 (2000) 1047.

[3] ITOH, S., et al., in Plasma Physics and Controlled Nuclear Fusion Research 1996 (Proc. 16th Int. Conf. Montreal, 1996), Vol.3, IAEA, Vienna (1997) 351.

[4] SAKAMOTO, M., et al., in Controlled Fusion and Plasma Physics (Proc. 24th Eur. Conf., 1997) Vol.21A, PartII, European Physical Society, Geneva (1997) 721.

[5] BEHRISCH, et al., J. Nucl. Mater. 233-237 (1996) 673.

[6] HIRAI, T., et al., J. Nucl. Mater. 258-263 (1998) 1060.

[7] Private communication, CHATELIER, M.

[8] HIRAI, T., et al., J. Plasma Fusion Res. SERIES (to be published). 